\title{
On numerical integration for effective stress assessment at notches
}

\author{
Enrico Maggiolini, Paolo Livieri \\ University of Ferrara (Italy) \\ Roberto Tovo \\ University of Ferrara (Italy)
}

\begin{abstract}
This paper presents a numerical method for non-local stress assessment by means of a general FE tool and the local stress field. Unlike usual calculations by means of a numerical PDE solver, a more general numerical integration is used. Different solutions are compared theoretically and numerically by evaluating the results obtained by two different FEM commercial software. The application of the non-local tension field is applied to the strength assessment of notches, welded joints and cracks.
\end{abstract}

KEYWORDS. Implicit gradient; Local tension field; Notches; Welding; Fatigue.

\section{INTRODUCTION}

$\mathrm{I}$ $\mathrm{n}$ real applications, it is usual for an engineer to deal with strength assessment at stress raisers, i.e. notches, welding or cracks. There are several theories and approaches explaining how to tackle such problems, but, sometimes, the proposed approaches are difficult to use. In particular, it is often impossible to apply the "non-local" effective stress values without a particular software tool because many procedures require the integration of a specific Partial Differential Equation to calculate the non-local stress field.

The goal of this research is to find a method for the evaluation of the non-local field in a fast and simple way, by using the local stress field available in all FEM software.

For this purpose, both Comsol Multiphysics and Ansys numerical software were used and compared.

Comsol has a pre-loaded module for an ordinary differential equation solution (Helmholtz equation) that can be used to calculate stress for non-local resistance, and even has an integration procedure for a general user-defined integral evaluation all-over the investigated domain. Alternatively, Ansys only has the possibility to compute and export the nodal coordinates and nodal results turned out from a conventional local structural analysis.

The final aim is to compute a non-local stress field by means of a sound conventional local stress analysis.

\section{IMPLICIT GRADIENT METHOD}

7 he authors recently presented a new way to estimate the fatigue life of notched structures and welded joints based on the Implicit Gradient [1,2].

This method is particularly suitable for a numerical estimation of the component fatigue life dependent on their geometry. The idea is very simple and enables application of the average damage originally formulated in the 1930s by Neuber [3], referred to as a geometry where fatigue crack propagation is on the bisector of the notch direction. This idea succeeds in attaining the fatigue limit of a plane component with notches by simple manual calculation [3,4]; on the other 
hand, it is difficult and awkward when the maximum stress or the crack initiation and propagation is outside the bisector or generally when the component is three dimensional or geometrically complex.

The implicit gradient method reinforces the idea that the damage should be related to the average of the stress components occurring on the body, where the values near to the critical point are more important than the far away field (by a weight function). The computed effective stress is representative of the overall damage in the process zone. The influence zone dimension is simply regulated by material properties and is indicated by the length $c$.

In a uniaxial fatigue case, the authors proposed to only average the first principal stress; for multi-axial fatigue it is necessary to use a multiaxial criterion, for instance, by using stress invariants or critical plane approaches, it is possible to define the ratio $\varrho$ between the hydrostatic component and the deviatoric component in the tension field averaging [5].

\section{MATHEMATICS OF THE PROBLEM}

I

$\mathrm{n}$ a body of volume $\mathrm{V}$, it is possible to define a non-local effective tension $\sigma_{\text {eff }}$ in a generic point $\mathrm{X}$ as an integral average of an equivalent local tension $\sigma_{\mathrm{eq}}$, weighted by a Gaussian function $\psi(\mathrm{x}, \mathrm{y})$ depending on the distance between points $\mathrm{x}$ and $\mathrm{y}$ of the body:

$$
\sigma_{\text {eff,int }}(\mathrm{x})=\frac{1}{\operatorname{Vr}(\mathrm{x})} \int_{\mathrm{V}} \psi(\mathrm{x}, \mathrm{y}) \sigma_{\mathrm{eq}}(\mathrm{y}) \mathrm{dy}=\frac{\int_{\mathrm{V}} \psi(\mathrm{x}, \mathrm{y}) \sigma_{\mathrm{eq}}(\mathrm{y}) \mathrm{dV}}{\int_{\mathrm{V}} \psi(\mathrm{x}, \mathrm{y}) \mathrm{dV}} \quad \text { in } \mathrm{V}
$$

where

$$
\psi=\frac{e^{\frac{-\|x-y\|^{2}}{2 \mathrm{~L}^{2}}}}{2 \mathrm{~L}^{2}}\left[\mathrm{~mm}^{-2}\right] \quad \mathrm{L}=\mathrm{c} \sqrt{2} \quad[\mathrm{~mm}]
$$

By approximating Eq. (1), it is possible to define an effective stress $\sigma_{\text {eff }}$ by the Helmholtz equation $[1,2,6]$ using Neuman boundary conditions $\left(\nabla \sigma_{\text {eff }} \cdot \mathrm{n}=0\right)[6]$.

$$
\sigma_{\text {eff }, \mathrm{IG}}-\mathrm{c}^{2} \nabla^{2} \sigma_{\text {eff }, \mathrm{IG}}=\sigma_{\mathrm{eq}} \quad \text { in } \mathrm{V}
$$

here $\sigma_{\mathrm{eq}}$ is the first principal stress and $\mathrm{c}$ is a material coefficient (for instance, it is $0.2 \mathrm{~mm}$ for weldable construction steel).

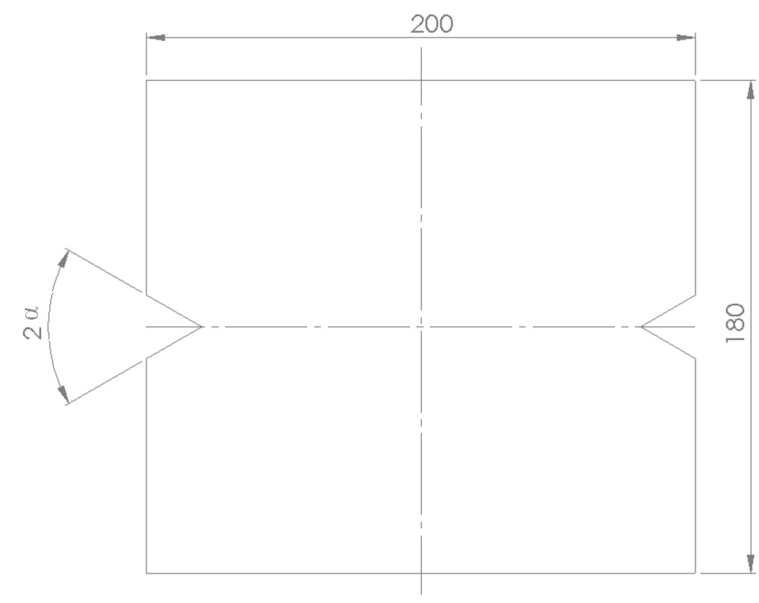

Figure 1: 2D geometry with a variable angle, size in $\mathrm{mm}$.

\section{ANALYSIS BY SOFTWARE WITH A BUILT-IN PDE SOLVER}

$\mathrm{T}$ he initial investigated geometry is a simple 2D geometry (Fig. 1), with the under linear elastic plane stress condition and remote tensile stress equal to one. The geometry has a notch-opening angle ranging from $0^{\circ}$ to $180^{\circ}$ and a notch tip radius varying from 0 to $1 \mathrm{~mm}$, the initially investigated parameter being the null radius. 
Comsol has a built-in PDE Solver, including the Helmholtz equation solver. The linear elastic stress solution provides the local stress field. The Helmholtz equation $\left(\sigma_{\text {eff,IG }}\right)$ can be directly solved on the same model.

One of the obtained outputs is given in Fig. 2. By changing the angle of the notch and plotting the results, it is possible to

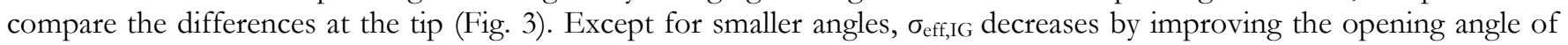
the notch.

Alternatively, by considering the integral value, the Eq. (1) can also be evaluated by the Comsol solver; Fig. 3 also shows such type of result $\left(\sigma_{\text {eff,int}}\right)$.

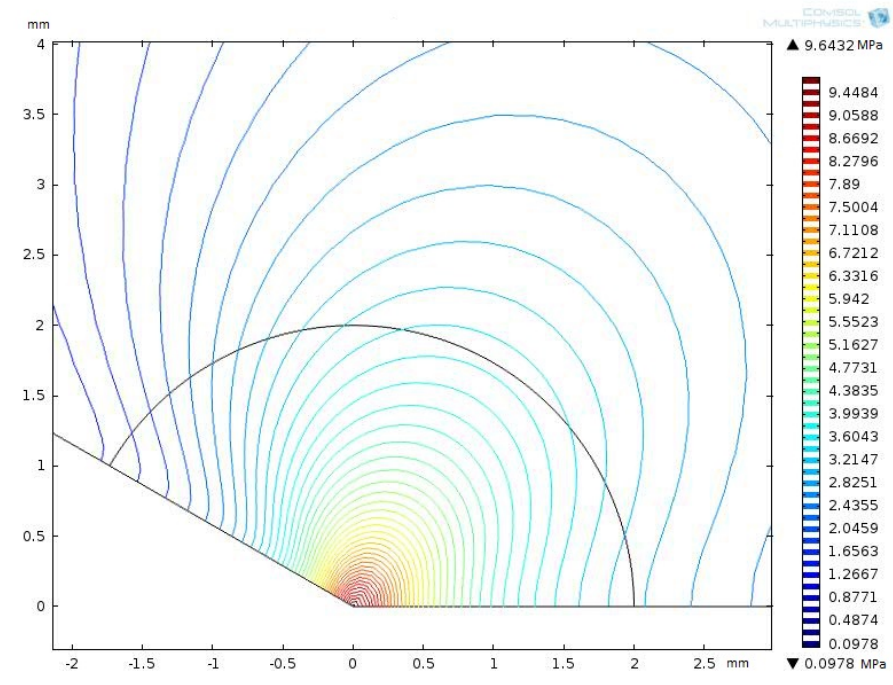

Figure 2: Example of plot of the $\sigma_{\text {eff,IG }}$

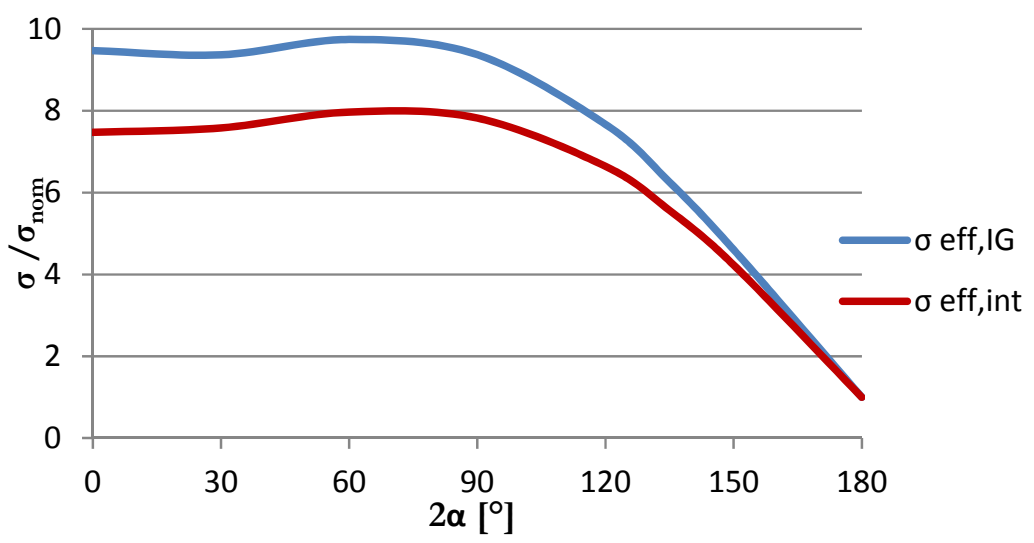

Figure 3: Trend of $\sigma_{\text {eff,IG }}$ VS $\sigma_{\text {eff,int }}$

\begin{tabular}{|c|c|c|c|}
\hline $2 \alpha$ & $\sigma_{\mathrm{eff}, \mathrm{IG}}$ & $\sigma_{\text {eff,int }}$ & Difference \\
\hline$\left[{ }^{\circ}\right]$ & [MPa] & {$[\mathrm{MPa}]$} & {$[\%]$} \\
\hline 0 & 9.47 & 7.47 & $-21.1 \%$ \\
\hline 30 & 9.37 & 7.58 & $-19.1 \%$ \\
\hline 60 & 9.74 & 7.97 & $-18.2 \%$ \\
\hline 90 & 9.37 & 7.82 & $-16.5 \%$ \\
\hline 120 & 7.67 & 6.64 & $-13.4 \%$ \\
\hline 135 & 6.26 & 5.56 & $-11.1 \%$ \\
\hline 150 & 4.61 & 4.23 & $-8.2 \%$ \\
\hline 180 & 1.00 & 1.00 & $0.0 \%$ \\
\hline
\end{tabular}

Table 1: Value of $\sigma_{\text {eff,IG }}$ and $\sigma_{\text {eff,int }}$ at any tested angle. 
To achieve a sufficient approximation, it is not necessary to compute the integral across the whole domain, but it is faster to only make the integration in a subdomain (a circular subdomain with a radius equal to $5.5 \mathrm{c}$ has been verified as suitable).

Differences between these two estimations can be assumed to be the intrinsic scatter between the two methods so that if the "integral" value is available, the "IG effective" value can be computed by this correction (Tab. 1).

\section{ANALYSIS BY SOFTWARE WITHOUT A BUILT-IN PDE SOLVER}

$\mathrm{M}$ ost FEM software does not have the PDE solver or integration option; nevertheless, it is possible to have a reasonable approximation by means of the knowledge of nodal coordinates and nodal local stress.

One possibility is to transform the integral into a summation, with a quadrature rules approximation [7]. For this calculation it is necessary to define a weight coefficient, called $\mathrm{N}$, built on the distance between nodes:

$$
\sigma_{\text {eff, int }}=\frac{\int_{\mathrm{V}} \psi(\mathrm{x}, \mathrm{y}) \sigma \mathrm{eq}(\mathrm{y}) \mathrm{dV}}{\int_{\mathrm{V}} \psi(\mathrm{x}, \mathrm{y}) \mathrm{dV}} \cong \frac{\sum \mathrm{N}(\mathrm{x}, \mathrm{y}) \psi(\mathrm{x}, \mathrm{y}) \sigma \mathrm{eq}(\mathrm{y})}{\sum \mathrm{N}(\mathrm{x}, \mathrm{y}) \psi(\mathrm{x}, \mathrm{y})}=\sigma_{\text {eff sum }} \quad \text { in } \mathrm{V}
$$

In a uniform mesh, i.e. with elements having the same dimension and constant distance between nodes, $\mathrm{N}$ should be constant. In any other case, $\mathrm{N}$ shall be computed depending on element dimension and actual node distances. This approach suggests using the nodal reaction (at each node constrained) as the $\mathrm{N}$ approximation, when the body is loaded by a uniform distributed load, such as the gravity load. Consequently, the bigger the element, the bigger the reaction.

Fig. 4 shows the nodal reaction in a subdomain in the case of a fairly regular, but not uniform, mesh.
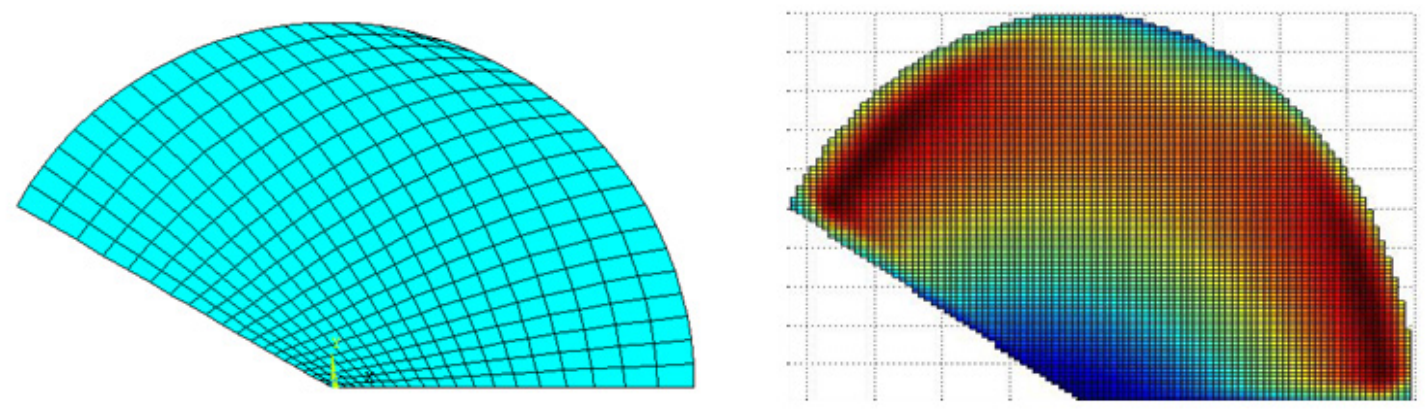

Figure 4: Plot of reaction $\mathrm{N}$ in a subdomain

For any kind of FE software, it is simply necessary to export coordinates of the node inside the subdomain, the first principal stress in each of those nodes, and the reaction of the same nodes. Any mathematical tool (i.e. Matlab or even a spreadsheet such as Excel) can compute $\sigma_{\text {eff,sum }}$ of Eq. (4).

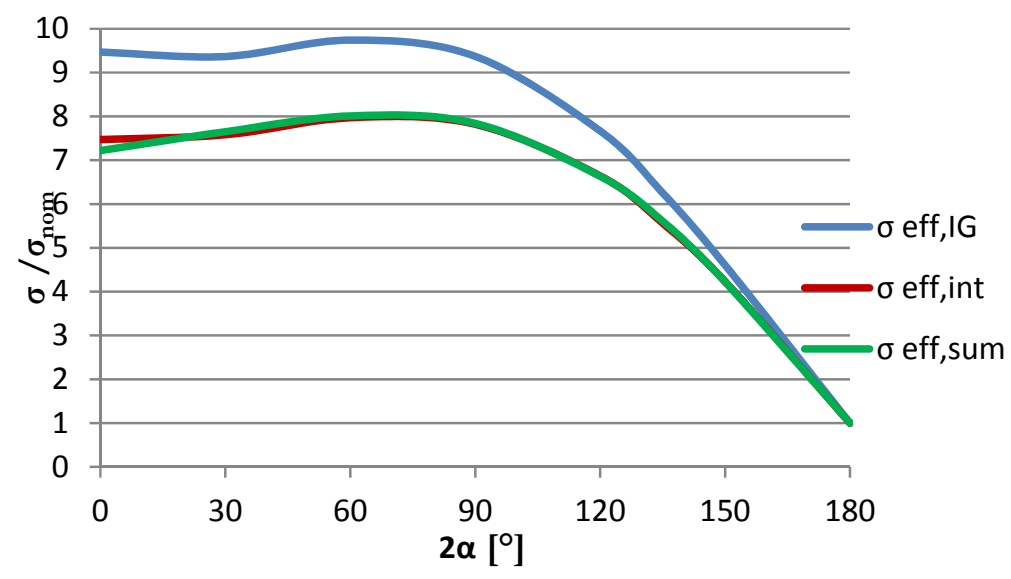

Figure 5: Trend of $\sigma_{\text {eff,int }}$ Vs $\sigma_{\text {eff,IG }}$ Vs $\sigma_{\text {eff,sum. }}$ 
The $\sigma_{\text {sum }}$ from the Ansys structural analysis is not so far from the Comsol integration for each opening angle taken into consideration (Fig. 5), it is never higher than $4 \%$ as a maximum error (Tab. 2).

\begin{tabular}{ccccc}
\hline $\begin{array}{c}2 \alpha \\
{\left[{ }^{\circ}\right]}\end{array}$ & $\begin{array}{c}\sigma_{\text {eff,IG }} \\
{[\mathrm{MPa}]}\end{array}$ & $\begin{array}{c}\sigma_{\text {eff,int }} \\
{[\mathrm{MPa}]}\end{array}$ & $\begin{array}{c}\sigma_{\text {eff,sum }} \\
{[\mathrm{MPa}]}\end{array}$ & $\begin{array}{c}\text { Error } \\
{[\%]}\end{array}$ \\
0 & 9.47 & 7.47 & 7.22 & -3.36 \\
30 & 9.37 & 7.58 & 7.65 & 0.91 \\
60 & 9.74 & 7.97 & 8.01 & 0.52 \\
90 & 9.37 & 7.82 & 7.84 & 0.26 \\
120 & 7.67 & 6.64 & 6.70 & 0.90 \\
135 & 6.26 & 5.56 & 5.61 & 0.85 \\
150 & 4.61 & 4.23 & 4.23 & -0.13 \\
180 & 1.00 & 1.00 & 1.00 & 0.00 \\
\hline
\end{tabular}

Table 2: Value of $\sigma_{\text {eff,IG }}, \sigma_{\text {eff,int }}$ and $\sigma_{\text {eff,sum }}$ at any tested angle.

\begin{tabular}{cccccc}
\hline $\mathrm{Q} / \mathrm{c}$ & $\begin{array}{c}\sigma_{\text {eff,IG }} \\
{[\mathrm{MPa}]}\end{array}$ & $\begin{array}{c}\sigma_{\text {eff,int }} \\
{[\mathrm{MPa}]}\end{array}$ & $\begin{array}{c}\text { Difference } \\
{[\%]}\end{array}$ & $\begin{array}{c}\sigma_{\text {eff,sum }} \\
{[\mathrm{MPa}]}\end{array}$ & $\begin{array}{c}\text { Error } \\
{[\%]}\end{array}$ \\
0 & 9.37 & 7.82 & $-16.5 \%$ & 7.84 & 0.26 \\
0.125 & 9.42 & 7.84 & $-16.8 \%$ & 7.79 & -0.60 \\
0.25 & 9.47 & 7.87 & $-16.9 \%$ & 7.87 & -0.03 \\
0.4 & 9.52 & 7.91 & $-16.9 \%$ & 7.91 & 0.01 \\
0.5 & 9.54 & 7.94 & $-16.8 \%$ & 7.94 & -0.05 \\
1 & 9.55 & 8.06 & $-15.6 \%$ & 8.06 & 0.00 \\
2 & 9.32 & 8.18 & $-12.2 \%$ & 8.18 & 0.00 \\
3 & 8.99 & 8.16 & $-9.2 \%$ & 8.16 & 0.00 \\
5 & 8.31 & 7.84 & $-5.6 \%$ & 7.84 & 0.04 \\
\hline
\end{tabular}

Table 3: Value of $\sigma_{\text {eff,IG }}, \sigma_{\text {eff,int }}$ and $\sigma_{\text {eff,sum }}$ at any tested ratio.

\section{CHANGING THE FILLED RADIUS}

$\mathrm{B}$ y modifying the ratio of the filled radius over c; it is possible to compute effective stress in rounded geometries as well. Results related to the opening-angle equal to $90^{\circ}$ are given in Tab. 3 and Fig. 6.

The scatter between the Implicit Gradient effective stress and the integral value decreases by increasing the notch tip radius. In any case, the error between the integral value and its numerical approximation is very low.

Hence, we can argue that the integral value of eq. (4) can be evaluated by means of generic FE software. The problem is the general relationship between the Implicit Gradient $\sigma_{\text {eff,IG }}$ and Integral value $\sigma_{\text {eff,int }}$ by combining different values of the opening angle and notch tip radius. The ratio between $\sigma_{\text {eff,IG }}$ and $\sigma_{\text {eff,int }}$ is given in Fig. 7.

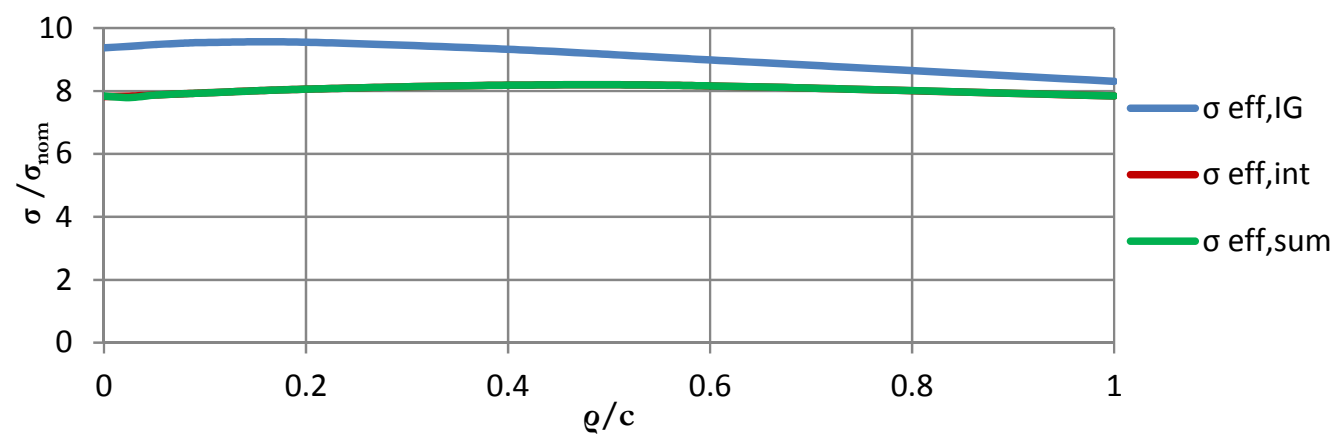

Figure 6: Trend of $\sigma_{\text {eff,IG }}$ Vs $\sigma_{\text {eff,int }}$ Vs $\sigma_{\text {eff,sum }}$ on the filled radius. 


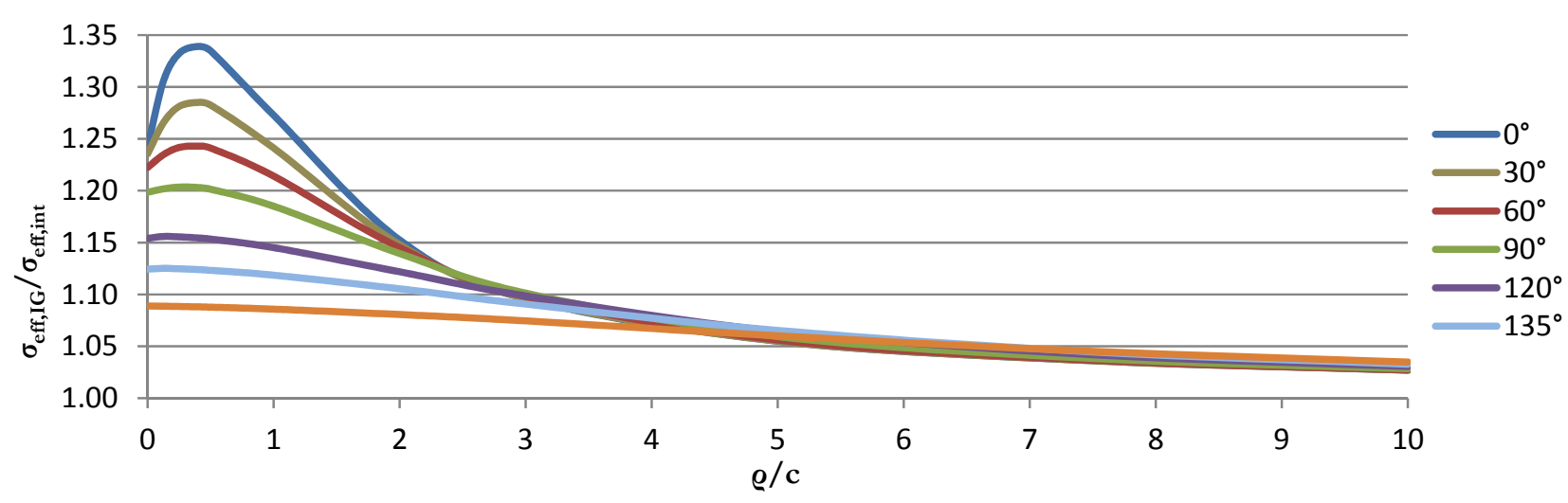

Figure 7: $\sigma_{\text {eff,IG }} / \sigma_{\text {eff,int }}$ function of filled radius and notch opening angle $2 \alpha$.

\section{MESH INFLUENCE}

A further problem regarding the numerical assessment of non-local effective stress is the sensibility of results to the elements dimension. Taking the $120^{\circ}$ geometry and through mesh analysis, it is possible to look at the relationship between the maximum element size and errors that occur. Tab. $4 \mathrm{~d}$ shows the dimension of the element at the notch tip. In this investigation "Plane 42" elements in Ansys and "quadrangular" elements in Comsol, were used.

\begin{tabular}{ccccc}
\hline Mesh & \multicolumn{2}{c}{ Comsol } & Ansys & Error \\
$\mathrm{d} / \mathrm{c}$ & $\sigma_{\text {eff,IG }}$ & $\sigma_{\text {eff,int }}$ & $\sigma_{\text {eff,sum }}$ & $\sigma_{\text {eff,int }}$ VS $\sigma_{\text {eff,sum }}$ \\
4 & 7.44 & 6.66 & 7.39 & $10.93 \%$ \\
3 & 7.53 & 6.67 & 7.58 & $13.74 \%$ \\
2 & 7.57 & 6.66 & 6.84 & $2.73 \%$ \\
1 & 7.65 & 6.65 & 6.70 & $0.76 \%$ \\
0.5 & 7.67 & 6.64 & 6.70 & $0.90 \%$ \\
0.25 & 7.67 & 6.64 & 6.70 & $0.96 \%$ \\
0.1 & 7.67 & 6.64 & 6.70 & $0.97 \%$ \\
\hline
\end{tabular}

Table 4: Error occurs in the mesh analysis.

\section{How To}

$\Lambda$ ccording to a previous theoretical framework, it is possible to sketch a procedure for effective non-local effective stress assessment by means of a general FE numerical tool. First of all, it is suitable to make a subdomain around the interested zone: this makes it easier to create a good quality mesh (element size close to c/2) and to export the needed results and no more. After that, it is only necessary to make an appropriate model and to export the nodal data: first principal stress of stress analysis, nodal coordinates, and reaction solutions under uniform loading. The Eq. (2) defines " $\psi$ " and the data are combined into the Eq. (4), $\mathrm{N}$ is the reaction solution and $\sigma_{\mathrm{eq}}$ is the first principal stress.

\section{THREE-DIMENSIONAL PROBLEM}

7 he applicability of the proposed approach was also verified for a three-dimensional component. The investigated structural detail is a welded joint depicted in Fig. 9. The obtained results are reported in Tab. 5. In this first application, the errors are slightly higher, however, it remains acceptable if used for design strength assessment. 

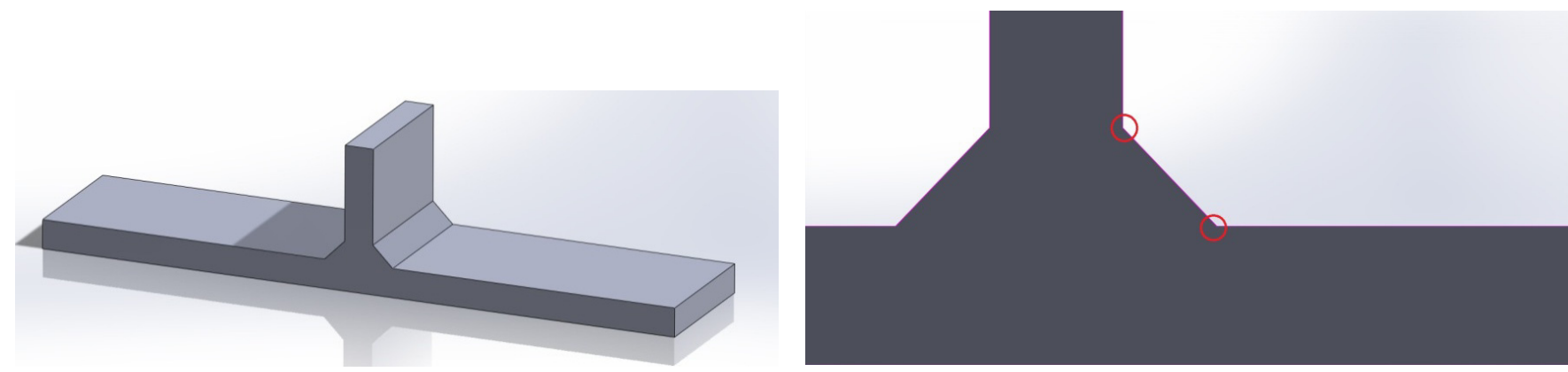

Figure 9: Three-dimensional geometry.

\begin{tabular}{ccccc}
\hline \multirow{2}{*}{ Dimension } & $\begin{array}{c}\sigma_{\text {eff,IG }} \\
{[\mathrm{MPa}]}\end{array}$ & $\begin{array}{c}\sigma_{\text {eff,int }} \\
{[\mathrm{MPa}]}\end{array}$ & $\begin{array}{c}\sigma_{\text {eff,sum }} \\
{[\mathrm{MPa}]}\end{array}$ & $\begin{array}{c}\text { Error } \\
{[\%]}\end{array}$ \\
$2 \mathrm{D}$ & 1.86 & 1.65 & 1.65 & -0.24 \\
$3 \mathrm{D}$ & 1.81 & 1.62 & 1.71 & 5.52 \\
\hline
\end{tabular}

Table 5: Comparison between 2D-3D of the same geometry.

\section{CONCLUSIONS}

$\mathrm{T}$ he Implicit Gradient method has already been demonstrated to be effective particularly in fatigue strength assessment of notches or joints. What was not available was a sound procedure for the effective stress evaluation in most FEM software, without integration or a PDE solver. With this method, through the definition of proper

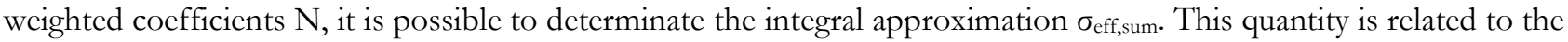
Implicit Gradient effective value and their relations depends on local geometry; such relationship has been investigated in this paper.

\section{REFERENCES}

[1] Tovo, R. Livieri, P., An implicit gradient application to fatigue of sharp notches and weldments, Engineering Fracture Mechanics, 74 (2007) 515-526.

[2] Tovo, R., Livieri, P., An implicit gradient application to fatigue of complex structures, Engineering Fracture Mechanics, 75(7) (2008) 1804-1814.

[3] Tanaka, K., Engineering formulae for fatigue strength reduction due to crack-like notches, International Journal of Fracture, 22 (1983) R39-R46.

[4] Livieri, P. Tovo, R., Fatigue limit evaluation of notches, small cracks and defects: an engineering approach, Fatigue and Fracture of Engineering Materials and Structures, 27 (2004) 1037-1049.

[5] Cristofori, A., Livieri, P., Tovo, R., An Application of the Implicit Gradient Method to welded structures under multiaxial fatigue loadings, International Journal of Fatigue, 31(1) (2009) 12-19.

[6] Peerlings, R.H.J., de Borst, R., Brekelmans, W.A.M., de Vree, J.H.P., Gradient enhanced damage for quasi brittle material, International Journal of Numerical Methods in Engineering, 39 (1996) 3391-3403.

[7] Dalquist, G., Björck, Å., Numerical Methods in Scientific Computing, Dover Publications, Inc., Mineola, New York, 1 (1974). 